\title{
Renormalization footprints in the phase diagram of the Grosse-Wulkenhaar model
}

\author{
Dragan Prekrat $\odot^{*}$ \\ Faculty of Physics, University of Belgrade, P.O. Box 44, SR-11001 Belgrade, Serbia
}

(Received 26 March 2021; revised 30 September 2021; accepted 2 November 2021; published 13 December 2021)

\begin{abstract}
We construct and analyze the phase diagram of a self-interacting matrix field in two dimensions coupled to the curvature of the noncommutative truncated Heisenberg space. In the infinite size limit, the model reduces to the renormalizable Grosse-Wulkenhaar model. The curvature term proves crucial for the diagram's structure. When turned off, the triple point collapses into the origin as matrices grow larger; when turned on, the triple point recedes from the origin proportionally to the coupling strength and the matrix size. The coupling attenuation that turns the Grosse-Wulkenhaar model into a renormalizable version of the $\phi_{\star}^{4}$ model cannot stop the triple point recession. As a result, the stripe phase escapes to infinity, removing the problems with UV/IR mixing.
\end{abstract}

DOI: $10.1103 /$ PhysRevD.104.114505

\section{INTRODUCTION}

Noncommutativity (NC) of space-time coordinates was initially proposed in the 1940s in the hope of resolving the confusion about the infinities in the nascent quantum field theory [1]. The first promising results in this regard were, however, achieved by the technique of renormalization. Since then, NC occasionally reemerged, both in the fundamental and the effective form, from condensed matter physics to quantum gravity [2,3]. Finally, when NC was discovered in the low energy sector of string theory at the turn of the millennium [4], various new NC models followed.

Contrary to the expected better-than-commutative behavior, NC models experience more difficulties with renormalizability. Generically, their nonplanar Feynman diagrams entangle small and large length scales, which prevents a successful absorption of divergences into the action terms [5-8]. It was shown that this UV/IR mixing could be resolved by the proper balancing of the scales provided by the Langman-Szabo duality [9].

The Grosse-Wulkenhaar (GW) model [10-12],

$S_{\mathrm{GW}}=\int \frac{1}{2}(\partial \phi)^{2}+\frac{\Omega^{2}}{2}\left(\left(\theta^{-1} x\right) \phi\right)^{2}+\frac{m^{2}}{2} \phi^{2}+\frac{\lambda}{4 !} \phi^{4}$,

*dprekrat@ipb.ac.rs

Published by the American Physical Society under the terms of the Creative Commons Attribution 4.0 International license. Further distribution of this work must maintain attribution to the author(s) and the published article's title, journal citation, and DOI. Funded by SCOAP ${ }^{3}$. managed to evade the UV/IR mixing problem. It features a self-interacting real scalar field on the Moyal space equipped with a $\star$ product,

$$
f \star g=f e^{i / 2 \grave{\partial} \theta \vec{\partial}} g \quad \Rightarrow \quad\left[x^{\mu}, x^{\nu}\right]_{\star}=i \theta^{\mu \nu} .
$$

Its potential is enhanced by the external harmonic oscillator term of a possible gravitational origin. Namely, the model can be reinterpreted [13] as that of a scalar field in a curved NC space of the truncated Heisenberg algebra $\mathfrak{h}^{\text {tr }}$,

$S_{\mathfrak{h}}=\int \sqrt{g}\left(\frac{1}{2}(\partial \phi)^{2}-\frac{\xi}{2} R_{\mathfrak{h}} \phi^{2}+\frac{m_{\mathfrak{h}}^{2}}{2} \phi^{2}+\frac{\lambda_{\mathfrak{h}}}{4 !} \phi^{4}\right)$.

The oscillator $\Omega$ term, which holds the key to renormalizability, is now seen as a coupling to the coordinatedependant curvature $R_{\mathfrak{h}}$. More details on the $\mathfrak{h}^{\text {tr }}$ and $S_{\mathfrak{h}}$ are provided later in the text. Another possible source of the oscillator term was presented in [14], where it elegantly appears in the expansion of the kinetic term of the free scalar field situated in the Snyder-de Sitter space. This model also predicts the running of the curvature coupling, which is an essential ingredient of the GW-mediated $\phi_{\star}^{4}$ renormalizability. It would be interesting to see if similar conclusions could be reached in the fuzzy de Sitter space $[15,16]$.

UV/IR mixing still poses a problem for gauge fields on NC spaces [17]. Hoping to build on the GW model's success, $[18,19]$ tried to adapt it to a gauge field on $\mathfrak{h}^{\text {tr }}$. Still, after extensive treatment, we found nonrenormalizability lurking in the form of divergent nonlocal derivative counterterms [20]. It turned out that, apart from the trivial vacuum, this model contains another, which breaks the 
translational invariance. This echoes the translational symmetry-breaking stripe phase that seems to be at the root of UV/IR mixing. Its "stripes" refer to patterns of spatially nonuniform magnetization, which appear when the field oscillates around different values at different points in space [21-23]. They also seem to shatter the symmetry between large and small scales that keeps the UV/IR mixing in check. Locally, the vacuum appears ordered, but globally, watched through the lenses of spatial averaging, it looks smudged into a disordered zero. It would be interesting to find out what happens with the stripe phase in the GW model. We would like to see how its renormalizability plays out from the phase transition point of view.

Phase diagrams on $\mathrm{NC}$ spaces have been extensively studied in various matrix models, since they regularize corresponding continuum theories in a numerical simulation-friendly fashion [24-44]. They generically feature three phases that meet at a triple point. Two of these are readily present in commutative theories; in the disordered phase, field eigenvalues clump around zero and in the ordered phase around one of the mirror-image minima of the potential. The third one is a matrix counterpart of the $\mathrm{NC}$ stripe phase; eigenvalues there gather both around positive and negative minima at the same time.

In [42], we started a numerical comparison of the twodimensional GW-model matrix regularization with $(R$-on) and without ( $R$-off) the curvature term, focusing mainly on the latter. Here, we present more details on the former and inspect how it bares under the oscillator term switching

$$
X=\frac{1}{\sqrt{2}}\left(\begin{array}{ccc} 
& \sqrt{1} & \\
\sqrt{1} & & \sqrt{2} \\
& \sqrt{2} & \ddots
\end{array}\right)_{N \times N}
$$

Identification [13] of $S_{\mathrm{GW}}$ and $S_{\mathfrak{h}}$ requires restriction to subspace $z=0$, which happens in a weak $N \rightarrow \infty$ limit of the matrix representation. This turns (4a) and (4b) into the Moyal space commutation relations.

The space $\mathfrak{h}^{\text {tr }}$ has a curvature,

$$
R_{\mathfrak{h}}=\frac{15 \mu^{2}}{2}-4 \epsilon \mu^{3} z-8 \epsilon^{2} \mu^{4}\left(x^{2}+y^{2}\right),
$$

which is represented (on $z=0$ ) by a negative diagonal matrix $R$ with elements,

$$
R_{i i}=\frac{31}{2}- \begin{cases}16 i, & i<N \\ 8 N, & i=N\end{cases}
$$

In its eigenvalues, we recognize energy levels of the harmonic oscillator. The quadratic dependence of $R_{\mathfrak{h}}$ on coordinates is precisely what we have in the $\Omega$ term in (1). off procedure that ensures the $\phi_{\star}^{4}$ model's renormalizability [10]. Moreover, since the triple point controls the extension of the problematic stripe phase, we try to pinpoint its location.

The paper is organized as follows. We first reintroduce the model and present its detailed $N=24$ phase diagrams. Then, we track the $R$-off triple point as we increase the matrix size. Finally, we present the effects of the curvature coupling variation on the phase diagram, look at the coupled model as we turn the coupling off, and compare its limit with the uncoupled one.

\section{MATRIX MODEL}

We here continue inspection of the matrix regularization $S_{N}$ of (3) started in [42]. Let us reintroduce the model and walk through its main features.

The coordinates $x, y$, and $z$ of the underlying $\mathfrak{h}^{\text {tr }}$ algebra satisfy

$$
\begin{aligned}
{[\mu x, \mu y] } & =i \epsilon(1-\mu z), \\
{[x, z] } & =+i \epsilon\{y, z\}, \quad[y, z]=-i \epsilon\{x, z\},
\end{aligned}
$$

where $\mu$ represents the mass scale and $\epsilon$ the strength of NC. If we set $\epsilon=1, \mu x$ and $\mu y$ can be represented by finitely truncated matrices of the Heisenberg algebra in the energy basis of the harmonic oscillator,

$Y=\frac{i}{\sqrt{2}}\left(\begin{array}{ccc} & -\sqrt{1} & \\ \sqrt{1} & & -\sqrt{2} \\ & \sqrt{2} & \ddots\end{array}\right)_{N \times N}$,

Derivatives in model (3), analyzed in the frame formalism, are realized as commutators $\partial_{\mu}=\left[p_{\mu}, \cdot\right]$ with momenta $p_{\mu}$,

$$
\frac{\epsilon p_{1}}{i \mu}=+\mu y, \quad \frac{\epsilon p_{2}}{i \mu}=-\mu x, \quad \frac{\epsilon p_{3}}{i \mu}=\mu z-\frac{1}{2} ;
$$

hence, their matrix counterparts are

$$
P_{1}=-Y, \quad P_{2}=X, \quad P_{3}=\frac{\mathbb{1}}{2} .
$$

The numbered identifications associate (3) with the matrix model,

$$
S_{N}=\operatorname{Tr}\left(\Phi \mathcal{K} \Phi-c_{r} R \Phi^{2}-c_{2} \Phi^{2}+c_{4} \Phi^{4}\right),
$$

in which the field $\Phi$ is a $N \times N$ Hermitian matrix and $\mathcal{K}$ the kinetic operator, 


$$
\mathcal{K} \Phi=\left[P_{\alpha},\left[P_{\alpha}, \Phi\right]\right]
$$

All originally dimensionful quantities are now expressed in units of $\mu$. We chose the minus sign of the mass term to enable positive $c_{2}$ to parametrize the relevant portion of the phase diagram, while positive $c_{4}$ ensures that $S_{N}$ is bounded from below. They are accompanied by the rescaled model parameters,

$$
\widetilde{c}_{2}=\frac{c_{2}}{N}, \quad \widetilde{c}_{4}=\frac{c_{4}}{N} .
$$

We performed parallel hybrid Monte Carlo simulations to measure various thermodynamic observables, most important being as follows:

(i) heat capacity, $C=\operatorname{Var} S / N^{2}$,

(ii) magnetic susceptibility, $\chi=\operatorname{Var}|\operatorname{Tr} \Phi| / N$,

(iii) distributions of eigenvalues and traces of $\Phi$,

where expectation value $\langle\mathcal{O}\rangle$ and variance $\operatorname{Var} \mathcal{O}$ of the observable $\mathcal{O}$ are given by

$$
\langle\mathcal{O}\rangle=\frac{\int \mathrm{d} \Phi \mathcal{O} e^{-S}}{\int \mathrm{d} \Phi e^{-S}}, \quad \operatorname{Var} \mathcal{O}=\left\langle\mathcal{O}^{2}\right\rangle-\left\langle\mathcal{O}^{2}\right\rangle
$$

Phase transitions in finite systems form smeared finite peaks and edges in profiles of free energy derivatives. Different quantities yield slightly different estimates of transition points, but they ultimately converge for large enough matrices. To locate them, we scanned through parameter space by varying $c_{2}$ at fixed $c_{4}$ - which played the role of the temperature - and searched for peaks in $C$ and $\chi$.

The classical equation of motion for $S_{N}$,

$$
2 \mathcal{K} \Phi-c_{r}\{R, \Phi\}+\Phi\left(-2 c_{2}+4 c_{4} \Phi^{2}\right)=0,
$$

gives us an idea what kind of phases to expect. Its kinetic, curvature, and pure potential parts are, respectively, solved by

$$
\Phi \propto \mathbb{1}, \quad \Phi=\mathbb{O}, \quad \Phi^{2}= \begin{cases}\mathbb{D}, & c_{2} \leq 0 \\ c_{2} / 2 c_{4} \mathbb{1}, & c_{2}>0\end{cases}
$$

corresponding to three phases depicted ${ }^{1}$ in Fig. 1:

(i) disordered $\uparrow$ phase: $\langle\Phi\rangle_{\uparrow}=\mathbb{O}$,

(ii) uniformly ordered $\uparrow \uparrow$ phase: $\langle\Phi\rangle_{\uparrow \uparrow} \propto \mathbb{1}$,

(iii) nonuniformly ordered $\uparrow \downarrow$ phase: $\langle\Phi\rangle_{\uparrow \downarrow} \propto U \mathbb{1}_{ \pm} U^{\dagger}$, where $U U^{\dagger}=U^{\dagger} U=\mathbb{1}, \mathbb{1}_{ \pm}^{2}=\mathbb{1}$ and $\left|\operatorname{Tr} \mathbb{1}_{ \pm}\right|<N$.

The $\uparrow \downarrow$ phase is a matrix equivalent of the stripe phase. A large mass parameter lives in the $\uparrow \uparrow$ phase and large quartic coupling in the $\uparrow$ phase, with the $\uparrow \downarrow$ phase nested in between. The phases meet at a triple point.

\footnotetext{
${ }^{1}$ Throughout this text, we use Wolfram Mathematica bluish StarryNightColors scheme for $R$-off plots and reddish SunsetColors scheme for $R$-on plots.
}
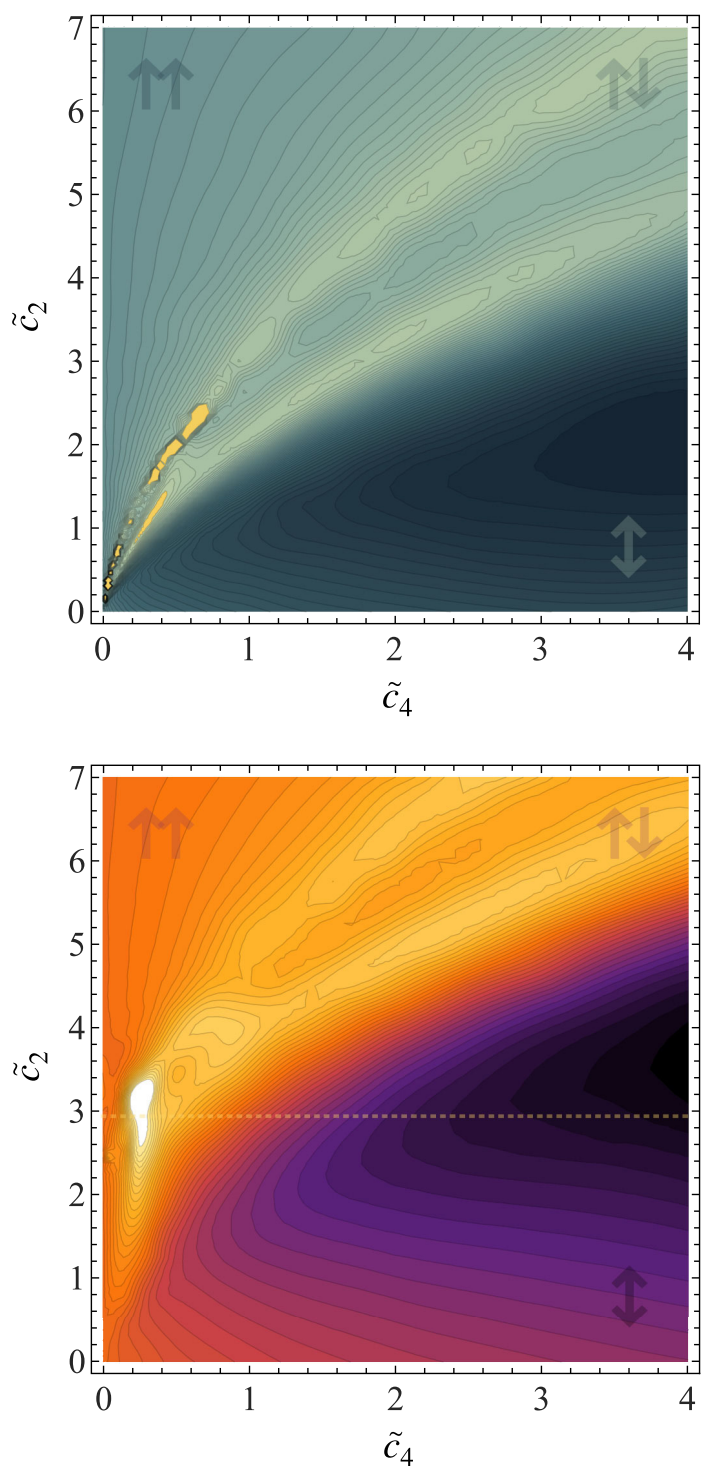

FIG. 1. Contour plots of $N=24$ phase diagram for $c_{r}=0$ (top) and for $c_{r}=0.2$ (bottom). Darker colors depict lower values and lighter colors higher values of specific heat, bright stripes being the transition lines. The dotted line on the bottom plot indicates a diagram shift relative to the $R$-off case. Phases are denoted by semitransparent arrows in the corners of the plot: $\uparrow$ phase occupies the bottom right, $\uparrow \uparrow$ phase the upper left, while the $\uparrow \downarrow$ phase is sandwiched in between, extending toward the upper right corner. Diagrams are constructed based on more than 5500 points.

When the kinetic term is negligible (e.g., field near $\propto \mathbb{1}$ ) and $c_{2} \geq \max _{i}\left\{c_{r}\left|R_{i i}\right|\right\}$, a diagonal solution exists that combines the effects of the curvature and the potential and which deforms the vacuum of the ordered phases,

$$
\Phi^{2}=\frac{c_{2} \mathbb{1}+c_{r} R}{2 c_{4}} .
$$

Both transitions out of the stripe phase in the $R$-off case (top plot in Fig. 1) follow the square root behavior for larger 
quartic coupling. For $N=24$ and $\widetilde{c}_{4}>1$, they are well approximated by

$$
\begin{aligned}
& \uparrow \rightarrow \uparrow \downarrow: \widetilde{c}_{2}=2.67(5) \sqrt{\widetilde{c}_{4}}-0.55(7), \\
& \uparrow \downarrow \rightarrow \uparrow \uparrow: \widetilde{c}_{2}=3.99(4) \sqrt{\widetilde{c}_{4}}-0.90(5) .
\end{aligned}
$$

For comparison, a pure potential model would show only a $\uparrow \rightarrow \uparrow \downarrow$ transition line $\widetilde{c}_{2}=2 \sqrt{\widetilde{c}_{4}}$ in the infinite $N$ limit.

In [42], we presented a simple argument that, due to diagonality, curvature acts as a quasimass term and should shift transition lines by $\delta c_{2}$ relative to the $R$-off case. The shift $\delta c_{2}$ is proportional to the $c_{r}$, and their ratio is bounded by

$$
\frac{1}{2 N} \leq \frac{\delta \widetilde{c}_{2}}{c_{r}} \leq 16-\frac{63}{2 N}
$$

We previously demonstrated this by numerical simulation at a token value of quartic coupling and with the absent kinetic term; here, we expose this effect in full in Fig. 1. Similar shifting is in the meantime also reported on the fuzzy sphere after adding a modification to the kinetic term [44]. The detailed analysis of the curvature's effects on the phase diagram is ongoing. It is presented elsewhere, while here we concentrate only on the aspects relevant to the position of the triple point.

We simultaneously inspect two finite limits of our matrix model, which zoom in on different portions of the parameter space,

$$
\begin{aligned}
& \mathcal{S}\left(c_{2}, c_{4}, c_{r}\right)=\lim _{N \rightarrow \infty} \frac{\left\langle S_{N}\left(c_{2}, c_{4}, c_{r}\right)\right\rangle}{N^{2}}, \\
& \widetilde{\mathcal{S}}\left(\widetilde{c}_{2}, \widetilde{c}_{4}, c_{r}\right)=\lim _{N \rightarrow \infty} \frac{\left\langle S_{N}\left(\widetilde{c}_{2}, \widetilde{c}_{4}, c_{r}\right)\right\rangle}{N^{2}} .
\end{aligned}
$$

In a way, phase diagram of $\widetilde{\mathcal{S}}$ describes the structure of the infinity of the phase diagram of $\mathcal{S}$. $\mathcal{S}_{0}$ and $\widetilde{\mathcal{S}}_{0}$ refer to $c_{r}=0$. We analyze $\mathcal{S}$ because it closely relates to $S_{\mathrm{GW}}$ up to a light adjustment of coefficients (Appendix A). We also complete the analysis of the $\widetilde{\mathcal{S}}_{0}$ started in [42], which tells us about the scaling properties of the third order $\uparrow \rightarrow \uparrow \downarrow$ transition line. Notice that parameters' mass dimensions are

$$
\left[c_{2}\right]=\left[c_{4}\right]=2, \quad\left[c_{r}\right]=0
$$

therefore, both $c_{2}$ and $c_{4}$ in $\widetilde{\mathcal{S}}$ are chosen to scale the same way with the momentum cutoff $\Lambda \sim \sqrt{N} \mu$, whereas $c_{r}$ does not scale at all.

\section{III. $R$-OFF TRIPLE POINT}

In the spirit of Bayesian probability notation, we write the coordinates $c_{i}$ of the triple point $T$ in the $R$-on and $R$-off case as $c_{i}(T \mid r)$ and $c_{i}\left(T \mid r^{\gamma}\right)$, respectively.

In [42], we found that the triple point of $\widetilde{\mathcal{S}}_{0}$ lies at $\widetilde{c}_{4}\left(T \mid r^{\gamma}\right) \lesssim 0.005$ [alternatively, $\quad c_{4}\left(T \mid r^{\gamma}\right) \lesssim 0.14$ from $N=28$ data] and established the descending trend of $\widetilde{c}_{4}\left(T \mid r^{\top}\right)$ with an increase in matrix size. In the meantime, we collected more data for matrix sizes up to $N=70$, allowing us to track the shrinking rate of the $\uparrow \rightarrow \uparrow \uparrow$ transition line. Unexpectedly, this transition disappears entirely, and the triple point collapses into the origin (Fig. 2).

Appendix B provides details on locating the triple point from raw data and different attempted data fits (Table I). We modeled small aberrations from the linear trend set by larger matrices by quadratic and power-law functions of $1 / N$. All the estimates agree with the triple point lying at the origin in the large $N$ limit, and the best one bounds its coordinates to

$$
\left(c_{2}, c_{4}\right)_{T} \leq(0.16,0.018),
$$

with a 95\% confidence level each, which is an order of magnitude improvement in precision.
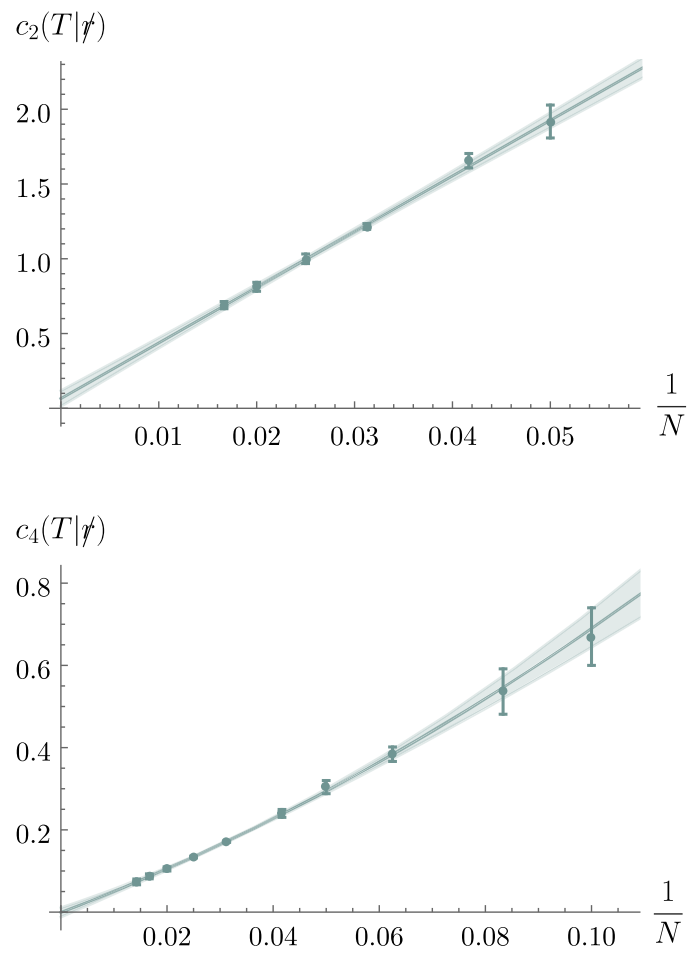

FIG. 2. Coordinates of the triple point in the $R$-off case as a function of the inverse matrix size. (Top) Linear fit of the $c_{2}$ coordinate: $c_{2}(T \mid \eta)=+0.07(5)+37(2) / N$. (Bottom) Quadratic fit of the $c_{4}$ coordinate: $c_{4}(T \mid \eta)=-0.000(12)+$ $4.9(7) / N+20(10) / N^{2}$. Data gathered from susceptibility $\chi$ for $N \leq 70$. The $\uparrow \rightarrow \uparrow \uparrow$ transition line ending in $T$ shrinks with an increase in matrix size and eventually disappears. 
In addition, linear extrapolation of the slopes of transition lines for $N=24,32,40,50$ shows that they radiate from the triple point/origin as

$$
\begin{aligned}
& \uparrow \rightarrow \uparrow \downarrow: c_{2}=7.1(8) c_{4}, \\
& \uparrow \downarrow \rightarrow \uparrow \uparrow: c_{2}=17(1) c_{4} .
\end{aligned}
$$

This is also how the phase diagram of $\widetilde{\mathcal{S}}_{0}$ looks like close to the origin, while away from it, its transition lines bend into $\sim \sqrt{\widetilde{c}_{4}}$.

It is important to notice that even if the triple point of $\mathcal{S}_{0}$ does not lie precisely at the origin, the triple point of $\widetilde{\mathcal{S}}_{0}$ will, due to $\widetilde{c}_{i}(T)=c_{i}(T) / N$. In fact, this holds for any alternate parameter rescaling $c_{i} / N^{\nu_{i}}$ by the positive power of the cutoff. This is in contrast with the $\phi^{4}$ model on the fuzzy sphere $[39,41]$. The culprit could be in differing forms of the kinetic term, whose presence allows the $\uparrow \rightarrow \uparrow \uparrow$ transition to develop in the first place.

\section{IV. $\boldsymbol{R}$-ON SHIFT AND RENORMALIZATION}

Coupling with curvature pushes the triple point-and with it the stripe phase-away from the origin proportionally to its strength (Fig. 3). The simulated shift of the triple point in the $N=24, R$-on case,

$$
\widetilde{c}_{2}(T \mid r)=0.18(8)+15.5(7) c_{r}
$$

relative to the $R$-off value,

$$
\widetilde{c}_{2}\left(T \mid r^{X}\right)=0.14(5)
$$

agrees well with the maximal prediction allowed by (18)

$$
\max \delta \widetilde{c}_{2}=\left(16-\frac{63}{2 N}\right) c_{r} \approx 14.7 c_{r} .
$$

The slight overshoot is discussed in Appendix B. Figure 2 shows that the small intercept of the $\widetilde{c}_{2}(T \mid r)$ line in Fig. 3 goes to zero with the increase in matrix size, so it is safe to assume that proportionality to $c_{r}$ becomes exact in the infinite size limit.

In the GW approach [10], renormalizability of the twodimensional $\phi_{\star}^{4}$ model is assured by defining it as a $\Omega \rightarrow 0$ limit of the series of superrenormalizable models in which $\Omega$ itself does not renormalize and serves as a series label [45]. It is chosen as

$$
\frac{1-\Omega^{2}}{1+\Omega^{2}}=\sqrt{1-\frac{1}{\left(1+\log \left(\Lambda / \Lambda_{R}\right)\right)^{2}}},
$$

which for large cutoff $\Lambda \sim \sqrt{N}$ switches off as

$$
\Omega \sim \frac{1}{\log N}
$$

Since Appendix A connects $\Omega$ and $c_{r}$ as

$$
c_{r}=\frac{\Omega^{2} / 8}{1-\Omega^{2} / 2},
$$

we consider the limit where $c_{r}$ decreases as

$$
c_{r} \sim \frac{1}{\log ^{2} N} .
$$

Combining this with $\delta \widetilde{c}_{2} \propto c_{r}$ (that is, $\delta c_{2} \propto N c_{r}$ ) would effectively swipe the stripe phase off to infinity as

$$
c_{2}(T) \sim \frac{N}{\log ^{2} N},
$$

leaving the limiting model with a completely different phase diagram from the one obtained by simply setting $c_{r}=0$.

Looking back at the equation of motion (14) and its solutions (15), we see that the curvature term prefers the trivial over striped vacuum. The action (10) also shows that the curvature itself compensates for the attenuation of the coupling. Namely, for nearly ordered field configurations
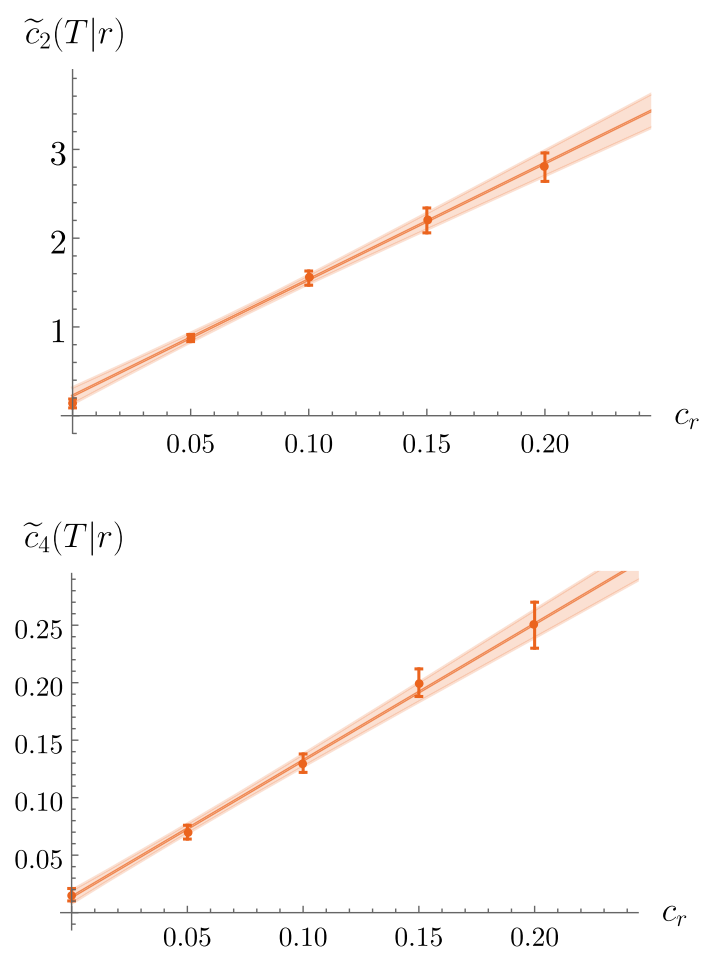

FIG. 3. Coordinates of the triple point in the $R$-on case as a function of the curvature coupling: $\tilde{c}_{2}(T \mid r)=0.18(8)+$ $15.5(7) c_{r}, \tilde{c}_{4}(T \mid r)=0.014(6)+1.19(8) c_{r}$. Data gathered from specific heat $C$ for $N=24$. 
$\Phi^{2} \propto \mathbb{1}$, the curvature term dominates the potential by the factor

$$
\frac{\operatorname{Tr} R \Phi^{2}}{\operatorname{Tr} \Phi^{2 n}} \approx \frac{\operatorname{Tr} R}{\operatorname{Tr} 1}=-8 N\left(1-\frac{31}{16 N}\right) \sim N
$$

which multiplied by the coupling leads once more to ratio

$$
\frac{N}{\log ^{2} N} \text {. }
$$

It is instructive to also track the behavior of the renormalized mass parameter as we turn off the curvature coupling. In [46], the divergent part of the mass renormalization in the $R$-on case is found to be

$$
\delta m_{\mathrm{ren}}^{2}=\frac{\lambda}{12 \pi\left(1+\Omega^{2}\right)} \log \frac{\Lambda^{2} \theta}{\Omega},
$$

which, adapted to our notation, gives the leading logarithmic mass divergence,

$$
\delta c_{2}^{\text {ren }} \sim-\log N .
$$

Bare $c_{2}$ has to compensate for this, increasing by $\left|\delta c_{2}^{\text {ren }}\right|$. Since this grows slower than (30), the bare $c_{2}$ required for the renormalization is located outside of the stripe phaseapparently, in the disordered phase.

This differs from the $R$-off case with $T$ at the origin. Although we cannot directly set $\Omega=0$ in (33), we could try to turn off $\Omega$ much faster than in the GW approach. Taking, for example,

$$
\Omega \sim e^{-N},
$$

would give the leading divergence,

$$
\delta m_{\text {ren }}^{2} \sim \lambda N,
$$

and

$$
\delta c_{2}(T) \sim N e^{-2 N} \rightarrow 0,
$$

as expected in the $R$-off case. Thus, a suitably chosen infinitesimal $\lambda \sim 1 / N$ would make the renormalization finite, leaving the bare mass inside the near-origin portion of the stripe phase for a range of physical mass choices in the perturbative regime.

\section{CONCLUDING REMARKS}

This paper aimed to see if the renormalizable GW redefinition of the $\phi_{\star}^{4}$ model is reflected in its corresponding phase diagram and if it affects the extent of the stripe phase connected to the UV/IR mixing. With that in mind, we tracked the triple point position; this is where the stripe phase starts, spreading toward larger values of the mass and quartic parameters. We compared the matrix regularization of the $\phi_{\star}^{4}$ model with the disappearing curvature term to the one without one, since its inclusion is crucial for the renormalizability.

We first refined the estimate [42] of the triple point position when the curvature term is turned off and concluded that it collapses into the origin in the infinite matrix size limit, completely removing the $\uparrow \rightarrow \uparrow \uparrow$ transition line. As the curvature coupling turns on, it shifts the triple point toward larger values of the mass parameter, proportionally to its strength and the matrix size. GW coupling attenuation does not reverse this effect. Instead, the triple point moves to infinity, erasing the stripe phase.

This leads to different phase diagrams for the renormalizable and nonrenormalizable version of the $\phi_{\star}^{4}$ model. The former seems to be stripe phase free, while in the latter the stripe phase is tethered to the origin of the parameter space. This demonstrates that GW construction affects both renormalizability and phase structure.

We are currently completing the exploration of the $R$-on model, and we also plan to further inspect the $R$-off triple point for $N>70$ to make sure it lies at the origin. Although we observed the convincing curvature-mediated linear shift for $N=24$ in the $R$-on case, it would be prudent to confirm the effect for larger matrices as well. We would also like to simultaneously decrease the coupling and increase the matrix size since their combined effect was here indirectly deduced.

Additionally, we mean to revisit the GW-inspired gauge model [20] in the hope of uncovering the reverse effect: nonrenormalizability due to retention of the stripe phase. After fixing the NC strength and scale, the model is left with only one adjustable parameter - the field strength coupling. Its additional stripelike vacuum transforms into a trivial one for the infinite coupling, implying the phase diagram's possible structure: stripe phase for weak interaction and disordered phase for strong interaction. This agrees with the phase diagrams in Fig. 1, where ordered phases lie at smaller quartic coupling compared to the disordered phase.

Another possible line of investigation could be the phase diagram of the renormalizable spinor model on $\mathfrak{h}^{\text {tr }}$ [47] in the context of fermionic matrix models $[48,49]$.

If this correspondence between renormalizability and phase structure proves to hold across models, it might be helpful when constructing new ones. For example, one could search numerically for early signatures of (non) renormalizability in their phase diagrams, assessing the new model's renormalizability potential, even before the involved and time-consuming analytical exploration.

\section{ACKNOWLEDGMENTS}

This work was supported by the Serbian Ministry of Education, Science and Technological Development Grant 
No. ON171031 and by COST Action MP1405 (ECOSTSTSM-MP1405-270217-081839, ECOST-STSM-MP1405160718-100016). The author would like to thank Maja Burić, Denjoe O'Connor, Samuel Kováčik, and Sebastián Franchino-Viñas for valuable discussions and DIAS for hospitality and financial support.

\section{APPENDIX A: MODEL CORRESPONDENCE}

According to [37], mapping

$$
\phi \leftrightarrow \Phi, \quad \int \leftrightarrow \sqrt{\operatorname{det} 2 \pi \theta} \operatorname{Tr}
$$

connects field theory on Moyal space and matrix field theory with the same parameters. Also, [13] provides a correspondence between $S_{\mathrm{GW}}$ and $S_{\mathfrak{h}}$,

$$
\begin{aligned}
S_{\mathrm{GW}} & =\left(1-\frac{\Omega^{2}}{2}\right) S_{\mathfrak{h}}, \\
m^{2} & =\left(1-\frac{\Omega^{2}}{2}\right)\left(m_{\mathfrak{h}}^{2}-\frac{15}{2} \xi \mu^{2}\right), \\
\lambda & =\left(1-\frac{\Omega^{2}}{2}\right) \lambda_{\mathfrak{h}}, \\
\Omega^{2} & =8 \epsilon^{2}\left(1-\frac{\Omega^{2}}{2}\right) \xi .
\end{aligned}
$$

From these, by comparing (3) and (10), it is easy to conclude that $S_{\mathrm{GW}}$ and $S_{N}$ are connected by

$$
\begin{aligned}
S_{\mathrm{GW}} & =\pi\left(1-\frac{\Omega^{2}}{2}\right) S_{N}, \\
m^{2} & =-\left(1-\frac{\Omega^{2}}{2}\right)\left(c_{2}+\frac{15}{2} c_{r}\right), \\
\lambda & =12\left(1-\frac{\Omega^{2}}{2}\right) c_{4}, \\
\Omega^{2} & =8\left(1-\frac{\Omega^{2}}{2}\right) c_{r},
\end{aligned}
$$

in the large $N$ limit $\left(\theta^{12}=1 / \mu^{2}\right.$, units: $\left.\mu=1\right)$.

Furthermore, an action multiplier can be absorbed into the field during expectation value integration and will affect only $c_{4}$,

$$
\begin{aligned}
\left\langle\kappa S\left(c_{2}, c_{4}, c_{r}\right)\right\rangle_{\kappa S} & =\left\langle S\left(c_{2}, c_{4} / \kappa, c_{r}\right)\right\rangle_{S}, \\
\sqrt{\kappa}\langle\Phi\rangle_{\kappa S}\left(c_{2}, c_{4}, c_{r}\right) & =\langle\Phi\rangle_{S}\left(c_{2}, c_{4} / \kappa, c_{r}\right),
\end{aligned}
$$

yielding

$$
\begin{aligned}
& C_{\kappa S}\left(c_{2}, c_{4}, c_{r}\right)=C_{S}\left(c_{2}, c_{4} / \kappa, c_{r}\right), \\
& \kappa \chi_{\kappa S}\left(c_{2}, c_{4}, c_{r}\right)=\chi_{S}\left(c_{2}, c_{4} / \kappa, c_{r}\right) .
\end{aligned}
$$

Since we are interested in the position of peaks of $C$ and $\chi$, this means that phase transition diagrams for $\kappa S$ and $S$ will be the same up to a reparametrization,

$$
\left(c_{2}, c_{4}, c_{r}\right) \leftrightarrow\left(c_{2}, c_{4} / \kappa, c_{r}\right) .
$$

For phase diagrams of $S_{\mathrm{GW}}$ and $\mathcal{S}$ in the $\Omega \rightarrow 0$ limit $\left(c_{r} \rightarrow 0\right)$, this means

$$
\left(m^{2}, \lambda\right) \leftrightarrow\left(-c_{2}, 12 c_{4} / \pi\right) .
$$

\section{APPENDIX B: TRIPLE POINT PROXIES AND FITS}

We here discuss triple point proxies used for Figs. 2 and 3.

The split of the profile of $\chi$ into two separate peaks as the $\uparrow \rightarrow \uparrow \uparrow$ line bifurcates into $\uparrow \rightarrow \uparrow \downarrow$ and $\uparrow \downarrow \rightarrow \uparrow \uparrow$ changes the slope of the $\uparrow \uparrow$-phase border $\partial_{\uparrow \uparrow}$ in Fig. 4. Therefore, the midpoint between the last point in the one-peak regime and the first point in the two-peak regime served as the triple point proxy for Fig. 2. We used the standard deviation of the triangular distribution ending at these two points as the triple point position uncertainty. For $N \leq 24$, the two peaks are not completely separated in the triple point region, so we instead took the intersection of extrapolated transition lines.

For consistency, we also checked the $C$ data, which has less predictive power due to larger uncertainties and distance from the triple point region. We there extrapolated the $\uparrow \rightarrow \uparrow \downarrow$ transition line to its intersection with $\partial_{\uparrow \uparrow}$. To get $68 \%$ confidence intervals of the intersection point coordinates, we used $83 \%$ confidence intervals of transition line fits, since the probability of a triple point belonging to their intersection is given by

$$
P\left(\uparrow \rightarrow \uparrow \downarrow \cap \partial_{\uparrow \uparrow}\right)=P(\uparrow \rightarrow \uparrow \downarrow) P\left(\partial_{\uparrow \uparrow}\right)
$$

and $0.68 \approx 0.83^{2}$.

In the $R$-on case, we used contour diagrams (e.g., Fig. 1) to detect the beginning of the $\uparrow \downarrow$ phase from $C$ data. We looked at the bright triple point peak position and then checked the neighboring raw data to pinpoint its exact location. As it turns out, the peak resolves into two very closely spaced convoluted peaks - which presumably coincide when matrix size increases-joined by a wall that separates phases (bottom of Fig. 5). For Fig. 3, we used the position of the protruding peak shown in Fig. 5, which gave a slightly higher estimate for the slope of the $c_{2}(T \mid r)=f\left(c_{r}\right)$ line than (25). The stationary point on this wall seems a more realistic estimator of the triple point position, but it is also more difficult to measure. A rough estimate using the stationary point, 

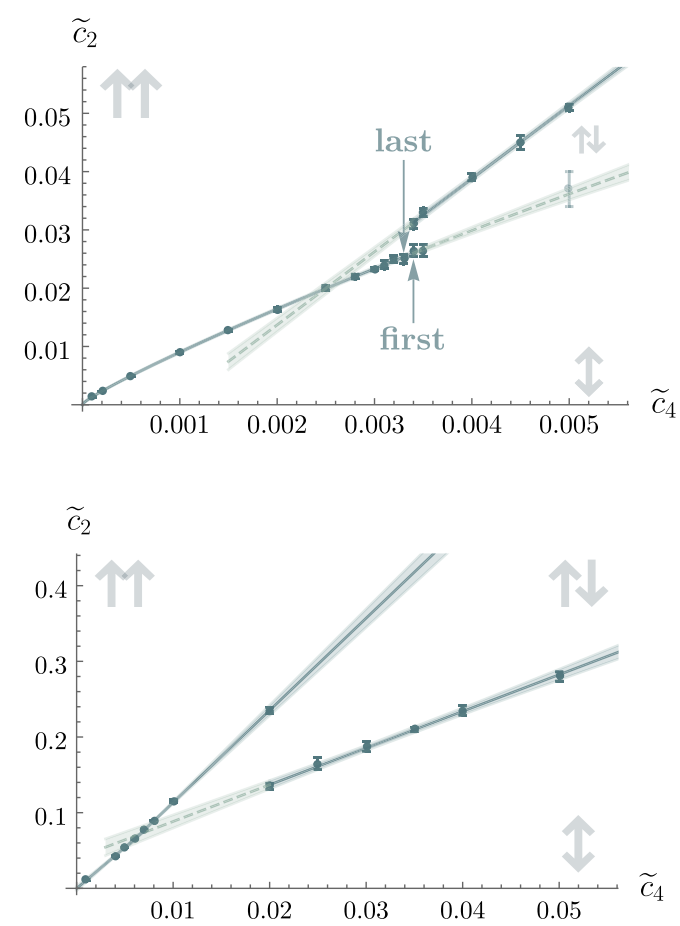

FIG. 4. Phase diagrams for $N=40$ in the vicinity of the triple point. (Top) Change in slope of the $\uparrow \uparrow$-phase border indicates a triple point. Arrows mark the last point on $\uparrow \rightarrow \uparrow \uparrow$ and the first point on $\uparrow \rightarrow \uparrow \downarrow$ lines. Constructed from $\chi$ data. (Bottom) Extrapolated transition lines with $83 \%$ confidence intervals. Constructed from $C$ data.

$$
\widetilde{c}_{2}(T \mid r)=13.2(11) c_{r}+0.24(9),
$$

fits within the interval (18) and is close to its upper bound. For comparison, the slope calculated from the smaller peaks is around 12 .

Looking at Fig. 1, we see a small oval local minimum region with a bright triple point peak at its lower left edge. Eigenvalue distribution there has the characteristics of the $\uparrow \downarrow$ phase. We do not believe this to constitute a separate
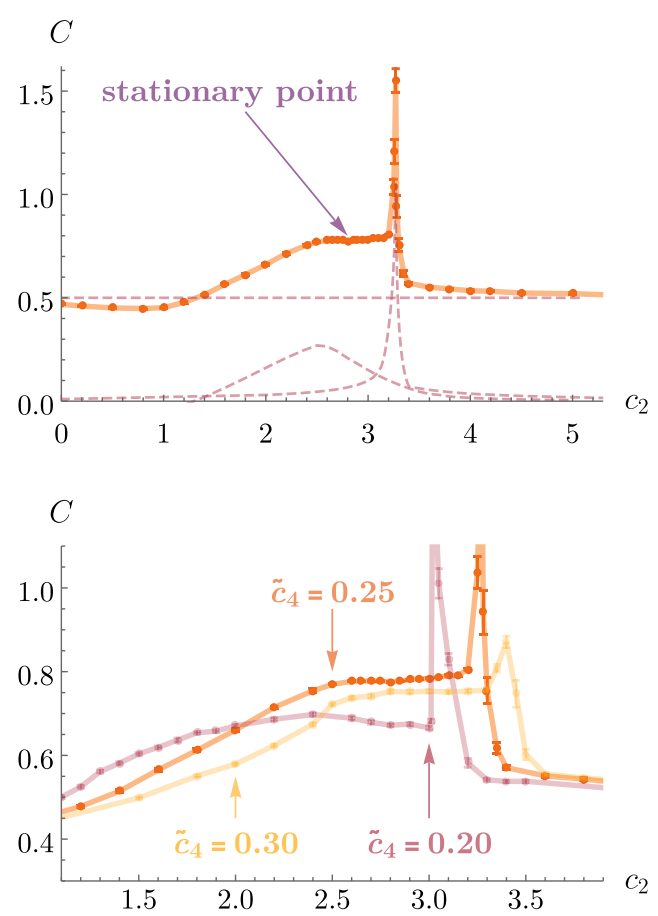

FIG. 5. (Top) Triple point region for $N=24, c_{r}=0.2$ at $\tilde{c}_{4}=$ 0.25 resolved into two peaks. The stationary point at the plateau is chosen as the triple point proxy. (Bottom) Plateau at $\tilde{c}_{4}=0.25$ mounts above plateaus at $\tilde{c}_{4}=0.20$ and $\tilde{c}_{4}=0.30$, building a wall between phases.

phase but a finite-size effect that collapses into a triple point as the matrix size increases. This should be, of course, checked at larger $N$. In addition, between the triple point and the origin in the $R$-on case, there is a transitional region where curvature eigenvalues in (16) slowly activate as we go from the $\uparrow$ to $\uparrow \uparrow$ phase. This might constitute a separate partially ordered phase but requires more data and further analysis.

Different extrapolations of the triple point position as a function of the inverse matrix size are collected in Table I.

TABLE I. Different models of $R$-off triple point position fitting. All intercepts are consistent with the triple point located at the origin. A linear fit is performed for data subsets with higher $N$, where nonlinearities are imperceptible.

\begin{tabular}{llll}
\hline \hline Data & Model & \multicolumn{1}{c}{$c_{4}(T \mid \eta)$ fit } & \multicolumn{1}{c}{$c_{2}(T \mid \eta)$ fit } \\
\hline$\chi$ & Linear & $c_{4}=-0.008(16)+5.7(7) / N$ & $c_{2}=+0.07(5)+37(2) / N$ \\
& Quadratic & $c_{4}=-0.000(11)+4.9(7) / N+20(10) / N^{2}$ & $c_{2}=+0.04(8)+39(5) / N-42(60) / N^{2}$ \\
& Power law & $c_{4}=+0.015(15)+12(4) / N^{1.24(11)}$ & $c_{2}=-0.0(2)+31(10) / N^{0.93(14)}$ \\
$C$ & Linear & $c_{4}=-0.12(10)+16(3) / N$ & $c_{2}=+0.9(9)+93(21) / N$ \\
\hline \hline
\end{tabular}


[1] H. S. Snyder, Quantized space-time, Phys. Rev. 71, 38 (1947).

[2] J. Bellissard, A. van Elst, and H. Schulz-Baldes, The noncommutative geometry of the quantum hall effect, J. Math. Phys. (N.Y.) 35, 5373 (1994).

[3] K. Fujii, From quantum optics to non-commutative geometry: A non-commutative version of the hopf bundle, veronese mapping and spin representation, arXiv:quant$\mathrm{ph} / 0502174$.

[4] N. Seiberg and E. Witten, String theory and noncommutative geometry, J. High Energy Phys. 09 (1999) 032.

[5] S. Minwalla, M. Van Raamsdonk, and N. Seiberg, Noncommutative perturbative dynamics, J. High Energy Phys. 02 (2000) 020.

[6] C.-S. Chu, J. Madore, and H. Steinacker, Scaling limits of the fuzzy sphere at one loop, J. High Energy Phys. 08 (2001) 038.

[7] B. P. Dolan, D. O'Connor, and P. Presnajder, Matrix $\phi^{4}$ models on the fuzzy sphere and their continuum limits, J. High Energy Phys. 03 (2002) 013.

[8] C. P. Martin, J. Trampetić, and J. You, UV/IR mixing in Noncommutative SU(N) Yang-Mills theory, Eur. Phys. J. C 81, 878 (2021).

[9] E. Langmann and R. J. Szabo, Duality in scalar field theory on noncommutative phase spaces, Phys. Lett. B 533, 168 (2002).

[10] H. Grosse and R. Wulkenhaar, Renormalization of $\phi^{4}$ theory on noncommutative $R^{2}$ in the matrix base, J. High Energy Phys. 12 (2003) 019.

[11] M. Disertori, R. Gurau, J. Magnen, and V. Rivasseau, Vanishing of beta function of non commutative $\phi_{4}^{4}$ theory to all orders, Phys. Lett. B 649, 95 (2007).

[12] Z. Wang, Constructive renormalization of the 2-dimensional Grosse-Wulkenhaar model, Ann. Henri Poincare 19, 2435 (2018).

[13] M. Burić and M. Wohlgenannt, Geometry of the GrosseWulkenhaar model, J. High Energy Phys. 03 (2010) 053.

[14] S. A. Franchino-Viñas and S. Mignemi, Asymptotic freedom for $\lambda \phi_{\star}^{4}$ QFT in Snyder-de Sitter space, Eur. Phys. J. C 80, 382 (2020).

[15] M. Burić, D. Latas, and L. Nenadović, Fuzzy de Sitter Space, Eur. Phys. J. C 78, 953 (2018).

[16] M. Burić and D. Latas, Discrete fuzzy de Sitter cosmology, Phys. Rev. D 100, 024053 (2019).

[17] D. N. Blaschke, Gauge fields on non-commutative spaces and renormalization, Fortschr. Phys. 62, 820 (2014).

[18] M. Burić, H. Grosse, and J. Madore, Gauge fields on noncommutative geometries with curvature, J. High Energy Phys. 07 (2010) 010.

[19] M. Burić, M. Dimitrijević, V. Radovanović, and M. Wohlgenannt, Quantization of a gauge theory on a curved noncommutative space, Phys. Rev. D 86, 105024 (2012).

[20] M. Burić, L. Nenadović, and D. Prekrat, One-loop structure of the U(1) gauge model on the truncated Heisenberg space, Eur. Phys. J. C 76, 672 (2016).

[21] S.S. Gubser and S. L. Sondhi, Phase structure of noncommutative scalar field theories, Nucl. Phys. B605, 395 (2001).
[22] P. Castorina and D. Zappala, Spontaneous breaking of translational invariance in non-commutative $\lambda \phi^{4}$ theory in two dimensions, Phys. Rev. D 77, 027703 (2008).

[23] H. Mejía-Díaz, W. Bietenholz, and M. Panero, The continuum phase diagram of the $2 \mathrm{~d}$ non-commutative $\lambda \phi^{4}$ model, J. High Energy Phys. 10 (2014) 056.

[24] D. J. Gross and E. Witten, Possible third order phase transition in the large $\mathrm{N}$ lattice gauge theory, Phys. Rev. D 21, 446 (1980).

[25] X. Martin, A matrix phase for the $\phi^{4}$ scalar field on the fuzzy sphere, J. High Energy Phys. 04 (2004) 077.

[26] M. Panero, Numerical simulations of a non-commutative theory: The scalar model on the fuzzy sphere, J. High Energy Phys. 05 (2007) 082.

[27] D. O'Connor and C. Saemann, Fuzzy scalar field theory as a multitrace matrix model, J. High Energy Phys. 08 (2007) 066.

[28] F. Garcia Flores, X. Martin, and D. O'Connor, Simulation of a scalar field on a fuzzy sphere, Int. J. Mod. Phys. A 24, 3917 (2009).

[29] F. Lizzi and B. Spisso, Noncommutative field theory: Numerical analysis with the fuzzy disc, Int. J. Mod. Phys. A 27, 1250137 (2012).

[30] A. P. Polychronakos, Effective action and phase transitions of scalar field on the fuzzy sphere, Phys. Rev. D 88, 065010 (2013).

[31] J. Tekel, Uniform order phase and phase diagram of scalar field theory on fuzzy $\mathbb{C} P^{n}$, J. High Energy Phys. 10 (2014) 144.

[32] B. Ydri, New algorithm and phase diagram of noncommutative $\phi^{4}$ on the fuzzy sphere, J. High Energy Phys. 03 (2014) 065.

[33] S. Rea and C. Sämann, The phase diagram of scalar field theory on the fuzzy disc, J. High Energy Phys. 11 (2015) 115.

[34] J. Tekel, Phase structure of fuzzy field theories and multitrace matrix models, Acta Phys. Slovaca 65, 369 (2015), http://www.physics.sk/aps/pub.php?y=2015\&pub=aps-1505.

[35] J. Tekel, Matrix model approximations of fuzzy scalar field theories and their phase diagrams, J. High Energy Phys. 12 (2015) 176.

[36] B. Ydri, K. Ramda, and A. Rouag, Phase diagrams of the multitrace quartic matrix models of noncommutative $\Phi^{4}$ theory, Phys. Rev. D 93, 065056 (2016).

[37] B. Ydri, Lectures on Matrix Field Theory (Springer, Cham, 2017).

[38] P. Sabella-Garnier, Time dependence of entanglement entropy on the fuzzy sphere, J. High Energy Phys. 08 (2017) 121.

[39] J. Tekel, Asymmetric hermitian matrix models and fuzzy field theory, Phys. Rev. D 97, 125018 (2018).

[40] K. Hatakeyama, A. Tsuchiya, and K. Yamashiro, Renormalization on the fuzzy sphere, Prog. Theor. Exp. Phys. 2018, 063B05 (2018).

[41] S. Kováčik and D. O'Connor, Triple point of a scalar field theory on a fuzzy sphere, J. High Energy Phys. 10 (2018) 010 . 
[42] D. Prekrat, K. N. Todorović-Vasović, and D. Ranković, Detecting scaling in phase transitions on the truncated Heisenberg algebra, J. High Energy Phys. 03 (2021) 197.

[43] M. Šubjaková and J. Tekel, Fuzzy field theories and related matrix models, Proc. Sci., CORFU2019 (2020) 189 [arXiv:2006.12605].

[44] M. Šubjaková and J. Tekel, Second moment fuzzy-field-theorylike matrix models, J. High Energy Phys. 06 (2020) 088.

[45] R. Wulkenhaar, Renormalisation of noncommutative $\phi_{4}^{4}$ theory to all orders (2004), https://ivv5hpp.uni-muenster.de/ u/raimar/publications/habilitation/raimar-habil.pdf.
[46] S. F. Viñas and P. Pisani, Worldline approach to the GrosseWulkenhaar model, J. High Energy Phys. 11 (2014) 087.

[47] M. Burić, J. Madore, and L. Nenadović, Spinors on a curved noncommutative space: Coupling to torsion and the GrossNeveu model, Classical Quantum Gravity 32, 185018 (2015).

[48] G. W. Semenoff and R. J. Szabo, Fermionic matrix models, Int. J. Mod. Phys. A 12, 2135 (1997).

[49] I. Aref'eva and I. Volovich, Spontaneous symmetry breaking in fermionic random matrix model, J. High Energy Phys. 10 (2019) 114. 